\title{
Comparison of Raman-scattering and Shubnikov-de Haas measurements to determine charge density in doped semiconductors
}

\author{
R. Cuscó, ${ }^{a)}$ L. Artús, and J. Ibáñez \\ Institut Jaume Almera, Consejo Superior d'Investigaciones Científicas (CSIC), Lluís Solé i Sabarís s.n., \\ 08028 Barcelona, Spain \\ N. Blanco and G. González-Díaz \\ Departamento de Física Aplicada III, Facultad de Física, Universidad Complutense, 28040 Madrid, Spain \\ M. Rahman and A. R. Long \\ Department of Physics and Astronomy, University of Glasgow, Glasgow G128QQ, Scotland
}

(Received 17 April 2000; accepted for publication 12 September 2000)

\begin{abstract}
We have verified the accuracy of free-charge determinations from Raman scattering in doped semiconductors by comparing the results obtained from phonon-plasmon coupled-mode line-shape fits with the charge-density values extracted from the analysis of the Shubnikov-de Haas oscillations. The experiments were carried out on $n$-InP layers, and conduction band nonparabolicity was included both in the Lindhard-Mermin model used to fit the Raman spectra and in the Shubnikov-de Haas analysis. We find a very good agreement between Raman and magnetotransport results, which confirms the reliability of the charge-density determination from Raman-scattering measurements when the line-shape analysis is carried out using the LindhardMermin model. (C) 2000 American Institute of Physics. [S0021-8979(00)02024-7]
\end{abstract}

\section{INTRODUCTION}

Raman scattering by LO phonon-plasmon coupled modes (LOPCMs) has been extensively used to study free carriers in semiconductors. ${ }^{1}$ While LOPCM Raman lineshape calculations are usually carried out using the fluctuation-dissipation theory, ${ }^{2,3}$ the electric susceptibility entering these calculations has been evaluated using different models, such as the Drude, the hydrodynamical, and the Lindhard-Mermin (LM) model. In some cases, Raman line shapes are calculated using the parameters obtained from electrical measurements, and then compared to the experimental Raman spectra. ${ }^{4,5}$ Some authors have obtained the carrier concentration just from the frequency of the $L^{+}$ coupled mode using the Drude model, ${ }^{6,7}$ and other authors have determined the damping parameter from the Drude model by fitting the Raman line shape with a fixed charge density determined by electrical measurements. ${ }^{8}$ Thus, only in a few Raman-scattering studies of LOPCMs in III-V compounds has the free-charge density been determined by actually fitting the calculated line shapes to the experimental Raman spectra. $^{9-15}$ Moreover, some of these studies were carried out using the Drude model, ${ }^{12,13}$ a simple LOPCM model which, as has been recently shown, ${ }^{16}$ does not yield accurate values of carrier concentration.

Charge density in doped semiconductors is customarily characterized by means of Hall-effect measurements. Thus, up to date the reliability of the charge-density values obtained from LOPCM analysis has only been checked against Hall data. ${ }^{7,15}$ On the other hand, these two techniques probe the free charge differently. Whereas Hall measurements yield

\footnotetext{
a) Author to whom correspondence should be addressed; electronic mail: rcusco@ija.csic.es
}

an average value of sheet resistance over the whole thickness of the doped layer, Raman scattering probes only a layer with a thickness on the order of the laser penetration depth, which for excitation wavelengths in the visible region is generally less than $100 \mathrm{~nm}$ in III-V semiconductors. Since the Hall coefficient is proportional to the thickness $d$ of the doped layer, uncertainties in the effective value of $d$ affect the values of charge density obtained from Hall measurements. Furthermore, carrier-density determinations by Hall measurements are affected by the Hall factor, which depends on the electron scattering mechanisms and on the temperature and therefore can vary for different samples and experimental measuring conditions.

We have shown recently ${ }^{16}$ that a LOPCM line shape model based on the LM dielectric function, in which band nonparabolicity has been included, gives very good fits to the Raman spectra and charge-density values in good agreement with the Hall-effect determinations. However, considering the differences between these two techniques discussed above and the necessary assumptions about the doped-layer thickness and the Hall factor made in the Hall-effect determination, checking the charge-density values obtained from Raman experiments with another independent technique becomes necessary to confirm their reliability.

At low temperature, the electron density can also be obtained from measurements of the Shubnikov-de Haas $(\mathrm{SdH})$ oscillations, whose periodicity allows the Fermi energy to be determined directly. This is the same parameter that is fitted in the Raman line-shape model based on the LM dielectric function, and its determination from the $\mathrm{SdH}$ periodicity is free from the uncertainties in the Hall scattering factor and doped-layer thickness that affect the Hall measurements. The aim of this article is to validate the accuracy of Raman scat- 


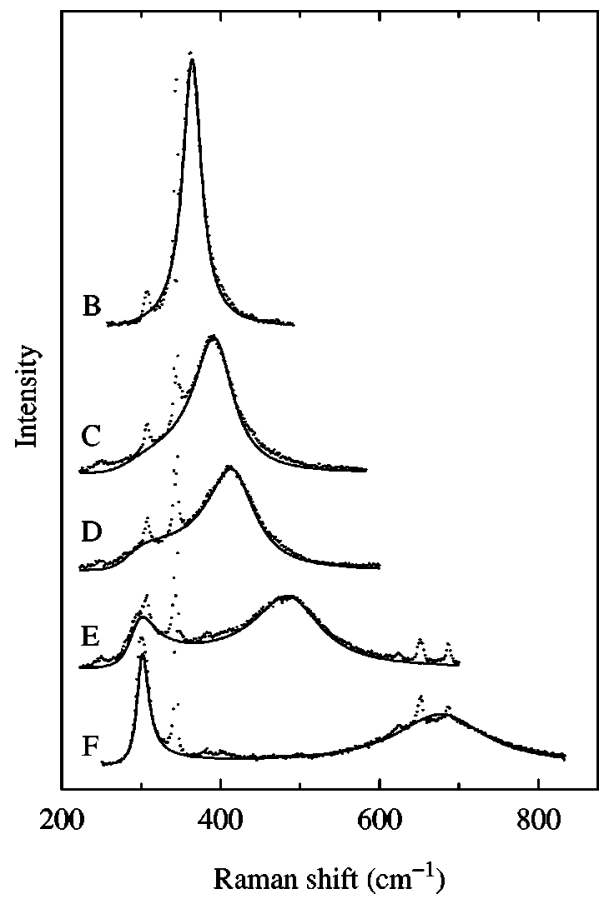

FIG. 1. B-F, fits of a LOPCM line-shape model based on the LindhardMermin dielectric function (solid line) to the room-temperature Raman spectra of $n$-InP samples (dots) with carrier densities of $3.7 \times 10^{17}, 6.6$ $\times 10^{17}, 8.4 \times 10^{17}, 1.4 \times 10^{18}$, and $3.5 \times 10^{18} \mathrm{~cm}^{-3}$, respectively.

tering to determine charge-density values from LOPCM lineshape fits by comparing them with the results obtained from the analysis of the $\mathrm{SdH}$ magnetotransport measurements.

\section{EXPERIMENT}

For this investigation we have used $n$-type InP layers obtained by $\mathrm{Si}^{+}$implantation on semi-insulating InP:Fe using a Greek-cross mask for a van der Pauw geometry. Double implantations followed by surface etching were carried out in order to obtain a homogeneous, flat doping profile that extends for $\approx 140 \mathrm{~nm}$ from the surface. Sample preparation details are described elsewhere. ${ }^{16}$ Ohmic contacts for low-temperature measurements were established by $\mathrm{Ni} / \mathrm{Au} /$ $\mathrm{Ge} / \mathrm{Au}$ evaporation and annealing at $380^{\circ} \mathrm{C}$ for $40 \mathrm{~s}$. The $\mathrm{SdH}$ measurements were carried out at $T=1.6 \mathrm{~K}$ in a Kelvinox $13 \mathrm{~T}$ superconducting magnet, using standard lock-in techniques. The Raman measurements were performed in a backscattering configuration on a (100) face using the 528.7 $\mathrm{nm}$ line of an $\mathrm{Ar}^{+}$laser as excitation. The Raman spectra were recorded at room temperature using a Jobin-Yvon T64000 spectrometer equipped with a charge coupled device detector cooled with liquid nitrogen.

\section{RESULTS AND DISCUSSION}

Figure 1 shows the LOPCM line-shape fits to the Raman spectra of several $n$-InP samples. The samples span a density range from $3.7 \times 10^{17}$ to $3.5 \times 10^{18} \mathrm{~cm}^{-3}$, and are labeled $\mathrm{B}$ to $\mathrm{F}$ for increasing doping level according to Ref. 16. For high carrier densities (D-F) both the $L^{+}$and $L^{-}$branches of the LOPCMs are observed in the Raman spectra, whereas for low carrier densities $(\mathrm{B}, \mathrm{C})$ only the $L^{+}$peak is detected. The
Raman peaks other than the LOPCMs that can be seen in Fig. 1, correspond to the forbidden TO mode and to zoneedge acoustic overtones, which give rise to a peak at $\approx 307$ $\mathrm{cm}^{-1},{ }^{16}$ the depletion zone LO mode observed at 343.5 $\mathrm{cm}^{-1},{ }^{16}$ and the characteristic second-order optical modes detected at 617,650 , and $682 \mathrm{~cm}^{-1} \cdot{ }^{17}$ Leaving aside the intensity of the LO mode, which depends on the extent of the depletion zone, these Raman features are independent of the doping level, and were subtracted prior to the LOPCM lineshape fits. As can be seen in Fig. 1 very good fits to the Raman spectra are obtained using a fluctuation-dissipation model in which the electric susceptibility is treated in the LM approximation. The details of the LOPCM line-shape model are given in Ref. 16. The use of the LM model, in which the undamped electric susceptibility is numerically evaluated from the Lindhard integral

$$
\begin{aligned}
\chi_{e}^{L}(q, \omega)= & \frac{e^{2}}{2 \pi^{3} q^{2}} \int f\left(E_{F}, T, k\right) \\
& \times\left(\frac{E(q+k)-E(k)}{[E(q+k)-E(k)]^{2}-(\hbar \omega)^{2}}\right) d^{3} k,
\end{aligned}
$$

where $f\left(E_{F}, T, k\right)$ is the Fermi distribution function for an electron gas with Fermi energy $E_{F}$ at temperature $T$, and $E(k)$ is the energy dispersion of the InP conduction band, allows nonparabolicity and finite temperature effects to be fully taken into account. The energy dispersion of the InP conduction band was calculated from a $14 \times 14 \mathbf{k} \cdot \mathbf{p}$ theory. ${ }^{18}$ Since we use Eq. (1) to calculate the electric susceptibility, the model fit yields the value of $E_{F}$, from which the electron density is obtained in terms of Fermi integrals by inverting an expansion of $E_{F}$ up to terms in $T^{7 / 2}$. As can be seen in Fig. 1 the use of the LM model, contrary to other simpler models such as the Drude or hydrodynamical approximations, allows us to obtain very good fits to the $L^{+}$and $L^{-}$ modes simultaneously. This fact, together with the high sensitivity to carrier concentration of the $L^{+}$mode, which exhibits frequency shifts of $\approx 320 \mathrm{~cm}^{-1}$ for electron-density variations of just about one order of magnitude, makes Raman scattering a very sensitive and powerful tool to determine charge density in doped InP.

To confirm the accuracy of the electron density values obtained from the LOPCM Raman line-shape fits discussed above, we have carried out magnetotransport measurements on the same set of $n$-InP samples. Figure 2(a) shows the magnetoresistance traces of samples B-F obtained at $1.6 \mathrm{~K}$, in which the nonoscillatory background resistivity has been subtracted by the method of the upper and lower envelopes. The oscillatory change of the resistivity with increasing magnetic field, the $\mathrm{SdH}$ effect, is due to the changing occupation of the Landau levels near the Fermi level, and therefore the periodicity of the oscillations provides a direct measure of the Fermi energy without having to make any assumptions about the values of the Hall scattering factor and the effective thickness of the implanted layer. Since most of the volume of the doped layer corresponds to the homogeneous core region near the surface, the periodicity of the $\mathrm{SdH}$ oscillations is determined by the electron density in this highdensity homogeneous region while, as we shall discuss be- 

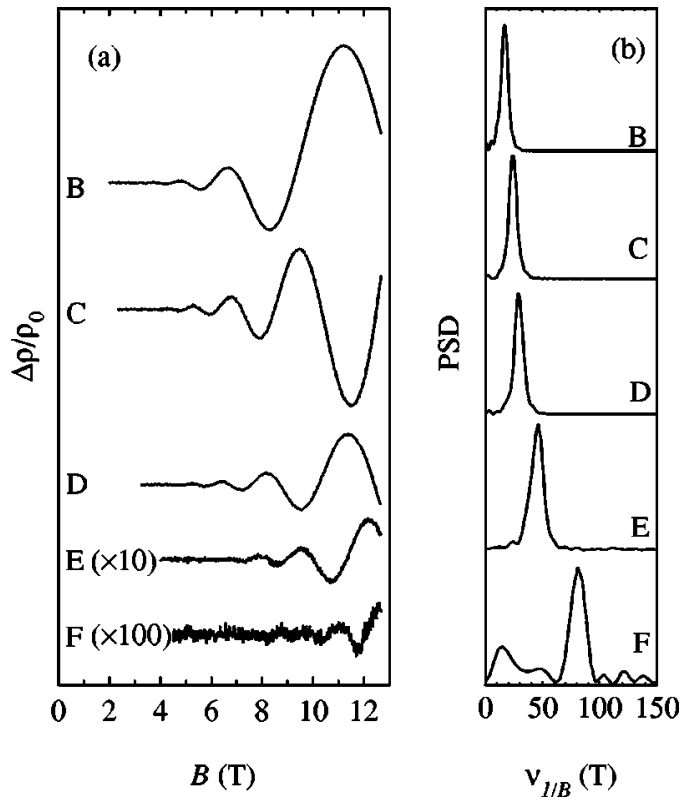

FIG. 2. (a) Magnetoresistance traces at $1.6 \mathrm{~K}$ corresponding to the $n$-InP samples whose Raman spectra are displayed in Fig. 1. The nonoscillatory background resistivity has been subtracted and the curves have been shifted vertically for clarity. (b) Arbitrarily normalized power spectral density (PSD) obtained from a Fourier transform of the magnetoresistance treated as a function of $1 / B$.

low, the far-end tail of the implantation profile is only reflected in a slight broadening of the oscillations. Therefore $\mathrm{SdH}$ and Raman measurements both probe the Fermi energy in the flat doping-profile region of the sample. The periodicity of the SdH oscillations for the transverse magnetoresistance is given by ${ }^{19}$

$$
\Delta\left(\frac{1}{B_{N}}\right)=\frac{e \hbar}{m^{*} E_{F}},
$$

where $B_{N}$ is the magnetic field at the extremum of the $N$ th oscillation, and $m^{*}$ is the cyclotron effective mass. The nonparabolicity of the InP electron effective mass and its dependence on carrier concentration have been studied by means of $\mathrm{SdH}$ measurements, ${ }^{20}$ and it has been shown that for carrier concentrations above $10^{18} \mathrm{~cm}^{-3}$ the cyclotron mass rapidly increases, in good agreement with $\mathbf{k} \cdot \mathbf{p}$ theory predictions. We have taken band nonparabolicity into account by using the band dispersion obtained from the $14 \times 14 \mathbf{~ k} \cdot \mathbf{p}$ calculation that was also used in the Raman line-shape model to calculate the cyclotron effective mass. The band-edge electron effective mass was taken as $m_{0}^{*}=0.076 m_{0} \cdot{ }^{20}$

We have obtained the periodicity of the $\mathrm{SdH}$ oscillations from the Fourier transform of the magnetoresistance treated as a function of $1 / B$ and also from plots of $1 / B_{N}$ versus quantum number. The magnetoresistance traces displayed in Fig. 2(a) show sinusoidal oscillations that can be closely fit using the theoretical $\mathrm{SdH}$ expression, ${ }^{19}$ and that reflect the electron density in the high-density homogeneously doped region near the surface. To confirm this point we have performed simulations of density profiles with a Gaussian density tail by adding up the contributions to the resistivity of the different layers of constant electron density across the

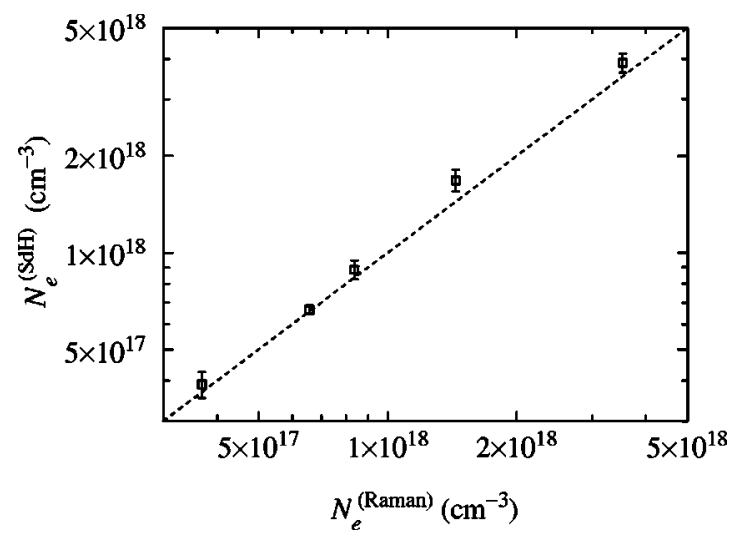

FIG. 3. Plot of the electron density values obtained from the periodicity of the Shubnikov-de Haas oscillations $\left(N_{e}^{\mathrm{SdH}}\right)$ vs the electron density values obtained from a line-shape fit to the Raman spectra using a LindhardMermin model $\left(N_{e}^{\mathrm{Raman}}\right)$. The dotted line corresponds to $N_{e}^{\mathrm{SdH}}=N_{e}^{\mathrm{Raman}}$ (total correlation).

doping profile. We find that the Fourier transform peak does not shift and the increase of its full width at half height is less than $6 \%$. This implies that the magnetoresistance oscillations are dominated by the contribution of the high-density homogeneous region, while the tail at the edge of the doping region, where electron density rapidly decreases, causes only a minor increase in the effective Dingle temperature and does not affect the deduced carrier density.

The range of carrier concentrations for which the $\mathrm{SdH}$ technique can be used is more limited than that of Raman spectroscopy. For highly doped samples, the rapid decrease of the lifetime $\tau$ of the electron states at the Fermi energy with increasing doping level smears out the $\mathrm{SdH}$ oscillations. The broadening of the Landau levels is characterized by the Dingle temperature $T_{D}=\hbar / 2 \pi k_{B} \tau$, which can be obtained from the analysis of the magnetic field dependence of the SdH oscillation amplitude. ${ }^{20} T_{D}$ is found to increase from 27 $\mathrm{K}$ for sample $\mathrm{B}$ to $45 \mathrm{~K}$ for sample $\mathrm{E}$ and $86 \mathrm{~K}$ for sample $\mathrm{F}$. This is reflected in the strong attenuation of the oscillations in samples $\mathrm{E}$ and $\mathrm{F}$ (see Fig. 2). For carrier concentrations higher than $5 \times 10^{18} \mathrm{~cm}^{-3}$, SdH oscillations are no longer visible within the limits of the magnetic field of our experimental setup. In contrast, being of semiclassical nature, the damping of LO-plasmon coupled modes is not so critical, and the plasmon-like $L^{+}$mode can be clearly observed for carrier densities as high as $10^{19} \mathrm{~cm}^{-3} \cdot{ }^{16}$ Conversely, for samples with very low doping concentration, the quantum limit is reached at moderate fields in the magnetotransport measurements, which precludes the unambiguous determination of the periodicity of the $\mathrm{SdH}$ oscillations. In this low carrier-density regime, Raman scattering can still be used to probe the charge density, although a correction for the photogenerated carriers may be necessary in some compounds. ${ }^{16,21}$

In Fig. 3 we show a correlation plot between the electron density values determined from the LM fits to the Raman spectra and those determined from the periodicity of the $\mathrm{SdH}$ oscillations. The error bars on the $\mathrm{SdH}$ data correspond to the width of the frequency bin in the fast Fourier transform used to determine the periodicity of the magnetoresistance oscil- 
lations. As can be seen in Fig. 3, we find a very good agreement between the values of electron density determined from Raman and magnetotransport experiments throughout the whole range of carrier concentrations in which both techniques can be compared.

\section{CONCLUSIONS}

The free-carrier density in doped semiconductors can be determined by means of optical and transport techniques involving different mechanisms. The analysis of the LOPCM Raman line shape based on the LM model allows the Fermi energy to be determined from fits to the experimental Raman spectra. On the other hand, magnetotransport measurements also probe the Fermi energy, which can be obtained from the periodicity of the Shubnikov-de Haas oscillations. We have applied these two techniques to evaluate the free-electron density of a set of $n$-doped InP samples, and we have included the nonparabolicity of the conduction band through a $14 \times 14 \mathbf{k} \cdot \mathbf{p}$ calculation in the analysis of both Raman and magnetotransport data. Using the LM model for the LOPCM line-shape analysis, a very good agreement between the carrier densities deduced from the magnetotransport measurements and the Raman-scattering determinations is obtained. These results constitute an independent and definite validation of the reliability of Raman scattering as a tool for contactless free-charge determinations in doped semiconductors over a wide range of doping concentrations.

\section{ACKNOWLEDGMENT}

The authors wish to acknowledge financial support from the Spanish Ministry of Science and Technology.
${ }^{1}$ G. Abstreiter, M. Cardona, and A. Pinczuk, in Light Scattering in Solids $I V$, edited by M. Cardona and G. Güntherodt, Topics in Applied Physics, Vol. 54 (Springer, Berlin, 1984).

${ }^{2}$ M. Cardona, in Light Scattering in Solids II, edited by M. Cardona and G. Güntherodt, Topics in Applied Physics, Vol. 50 (Springer, Berlin, 1982).

${ }^{3}$ D. T. Hon and W. L. Faust, Appl. Phys. (Berlin) 1, 241 (1973).

${ }^{4}$ G. Irmer, V. V. Toporov, B. H. Bairamov, and J. Monecke, Phys. Status Solidi B 119, 595 (1983).

${ }^{5}$ G. Irmer, M. Wenzel, and J. Monecke, Phys. Rev. B 56, 9524 (1997).

${ }^{6}$ D. J. Olego and H. B. Serreze, J. Appl. Phys. 58, 1979 (1985).

${ }^{7}$ B. Boudart, B. Prévot, and C. Schwab, Appl. Surf. Sci. 50, 295 (1991).

${ }^{8}$ R. Fukasawa and S. Perkovitz, Phys. Rev. B 50, 14119 (1994).

${ }^{9}$ G. Abstreiter, R. Trommer, M. Cardona, and A. Pinczuk, Solid State Commun. 30, 703 (1979).

${ }^{10}$ D. Olego and M. Cardona, Phys. Rev. B 24, 7217 (1981).

${ }^{11}$ W. Richter, U. Nowak, H. Jürgensen, and U. Rössler, Solid State Commun. 67, 199 (1988).

${ }^{12}$ K. Wan, J. F. Young, R. L. S. Devine, W. T. Moore, A. J. SpringThorpe, C. J. Miner, and P. Mandeville, J. Appl. Phys. 63, 5598 (1988).

${ }^{13}$ A. Mlayah, R. Carles, G. Landa, E. Bedel, and A. Muñoz-Yagüe, J. Appl. Phys. 69, 4064 (1991).

${ }^{14}$ K. Wan and J. F. Young, Phys. Rev. B 41, 10772 (1990).

${ }^{15}$ M. Ramsteiner, J. Wagner, P. Hiesinger, K. Köhler, and U. Rössler, J. Appl. Phys. 73, 5023 (1993).

${ }^{16}$ L. Artús, R. Cuscó, J. Ibáñez, N. Blanco, and G. González-Díaz, Phys. Rev. B 60, 5456 (1999).

${ }^{17}$ L. Artús, R. Cuscó, J. M. Martin, and G. González-Díaz, Phys. Rev. B 50, 11552 (1994).

${ }^{18}$ U. Rössler, Solid State Commun. 49, 943 (1984).

${ }^{19}$ B. K. Ridley, Quantum Processes in Semiconductors (Clarendon, Oxford, 1988).

${ }^{20}$ D. Schneider, D. Rürup, A. Plichta, H.-U. Grubert, A. Schlachetzki, and K. Hansen, Z. Phys. B: Condens. Matter 95, 281 (1994).

${ }^{21}$ R. Cuscó, J. Ibáñez, and L. Artús, Phys. Rev. B 57, 12197 (1998). 